\title{
Recent progress in angle-insensitive narrowband and broadband metamaterial absorbers
}

\author{
Saptarshi Ghosh, Toan Trung Nguyen, and Sungjoon Lim* \\ School of Electrical and Electronics Engineering, Chung-Ang University, Seoul, Republic of Korea
}

Received: 20 September 2018 / Accepted: 3 February 2019

\begin{abstract}
Recent progress in angle-insensitive narrowband and broadband metamaterial absorbers is presented herein. Initially, a few narrowband structures are described along with their absorption mechanisms. A bandwidth-enhanced absorber, conceptually derived from the existing narrowband geometry, is also discussed. Finally, several broadband absorbers having wide absorption bandwidths across different microwave frequency ranges are illustrated. The reported structures are primarily designed to exhibit high angularly stable responses suitable for practical applications. Furthermore, their geometries are fourfold symmetric, thereby displaying polarization-independent characteristics. Experimental verifications of the designed absorbers have been confirmed under normal and oblique incidences. The angle insensitivity, polarization independence, flexible absorption bandwidths (from narrowband to broadband), and commercial feasibility of the reported structures might establish them as potential candidates for manifold absorber applications.
\end{abstract}

Keywords: Metamaterials / electromagnetic absorbers / angle insensitivity / broadband absorbers

\section{Introduction}

Electromagnetic (EM) metamaterials (MMs) are artificial composite structures engineered to exhibit unique features not available in natural materials $[1,2]$. Owing to their properties in the subwavelength scale, MMs can manipulate the effective permittivity and permeability parameters in a wide range, from very small values to extremely large values, and even negative values in some cases [3-5]. Therefore, MMs have been exploited in various applications, e.g., super lens [6], antennas [7], filters [8], and cloaking [9]. The MM absorber is another breakthrough in this domain [10] that can be used as a potential alternative to conventional absorbers owing to their near-unity absorption properties, ultrathin thickness, simple manufacturing procedures, and increasing effectiveness.

Numerous absorber applications are available across civil and defense sectors. The reduction in the radar cross section is a crucial application [11], where the target is coated with microwave absorbers to reduce the detectability of the object by radar. In household applications, various electrical instruments need to be shielded to avoid any EM interference [12]. Other typical applications

\footnotetext{
* e-mail: sungjoon@cau.ac.kr
}

include solar cells [13], photodetectors [14], sensors [15], thermal emitters [16], and bolometers [17]. Some of these applications require narrowband absorptions (bolometers, sensors), whereas others use broadband absorbers covering wide bandwidths (stealth technology). As MM absorbers are built on large periodic arrays, their absorption responses depend on the incident as well as on the polarization angles of the incident EM wave [18]. However, practical applications require such kind of absorber structures whose performances will remain constant over any incident and/or polarization angle. Therefore, microwave absorbers having angular and polarization angle stability characteristics are urgently required in the research fraternity.

Herein, a recent progress in angle- and polarizationindependent MM absorbers is discussed in detail. In the initial sections, various narrowband structures are described along with their absorption mechanisms. Later, broadband absorbers are presented based on the resistive concept. All the geometries are fourfold symmetric in nature, thereby resulting in polarization-insensitive characteristics. The structures are further designed to exhibit higher angularly stable responses as compared to the existing absorbers. Finally, a summary of the absorber structures is presented in the tabular format, along with the possible advancements in the near future. 


\section{Absorption mechanism of metamaterial absorbers}

An MM absorber is typically built on a commercially available dielectric substrate, on which periodic arrays of conductive patterns are imprinted. The top layer geometry exerts an effective inductance and an effective capacitance, whereas the loss is attributed to the substrate. Depending on the pattern geometry and dielectric properties, single or multiple narrowband/broadband absorption responses can be obtained from the structure.

In 2008, Landy et al. [10] experimentally demonstrated a narrowband MM absorber, based on the combination of an electric ring resonator and a cut wire. The two asymmetrical patterns are periodically etched on the opposite sides of an FR4 substrate. When the plane EM wave is incident on the geometry, the design is excited such that both electric and magnetic resonances are generated. This simultaneously reduces the reflection as well as transmission coefficients of the structure and exhibits a narrowband absorption at $11.65 \mathrm{GHz}$. The proposed technique has several significant advantages over the conventional microwave absorbers such as thin profile, ease of fabrication, commercial availability, and practical effectiveness.

Henceforth, researchers have been actively investigating various types of MM absorbers that exhibit diverse characteristics. With the evolution in design principles, the rear side of the absorber structures has been shielded with complete metal plates $[19,20]$. This has led to the elimination of transmission responses from the geometries, and the subsequent absorbers are primarily emphasized in reducing the reflection coefficient only from the designs.

The absorptivity of a structure can be characterized as follows: $A=1-\left|S_{11}\right|^{2}-\left|S_{21}\right|^{2}$, where $S_{11}$ and $S_{21}$ are the reflection and transmission coefficients, respectively. Because the MM absorbers are of subwavelength-scaled geometries, other phenomena such as scattering and diffraction are too insignificant to be considered. With the complete ground plane, the transmission is zero; thus, the equation above is reduced to $A=1-\left|S_{11}\right|^{2}$. Thus, the absorptivity of a structure can be maximized through minimizing the reflection from the geometry.

The reflection coefficient at the interface is related to the input impedance of the structure according to the following equation [21]:

$$
S_{11}=\left(Z_{\text {in }}-Z_{0}\right) /\left(Z_{\text {in }}+Z_{0}\right),
$$

where $Z_{\text {in }}$ and $Z_{0}$ are the input impedance of the structure and free space impedance, respectively. It is clearly observed that the reflection coefficient becomes zero when $Z_{\text {in }}=Z_{0}$, i.e., when the structure is perfectly impedance matched to the free space. In summary, a structure will exhibit near-unity absorption if its input impedance is matched to the free space impedance (and no transmission at the back). Narrowband or broadband absorptions occur based on the nature of impedance matching of the geometry: matching at one frequency causes narrowband absorption, whereas matching over a wide frequency range results in broadband absorption.
However, the equation above is valid for normal incidences only. Under an oblique incidence, the reflection coefficient equation changes for perpendicular polarization $\left(\Gamma_{\perp}\right)$ and parallel polarization $\left(\Gamma_{\|}\right)$, as given by [21]

$$
\begin{gathered}
\Gamma_{\perp}=\frac{Z_{\text {in }} \cos \theta_{i}-Z_{0} \cos \theta_{t}}{Z_{\text {in }} \cos \theta_{i}+Z_{0} \cos \theta_{t}}, \\
\Gamma_{\|}=\frac{Z_{\text {in }} \cos \theta_{t}-Z_{0} \cos \theta_{i}}{Z_{\text {in }} \cos \theta_{t}+Z_{0} \cos \theta_{i}},
\end{gathered}
$$

where $\theta_{i}$ and $\theta_{t}$ are the incident and transmission angles, respectively. Therefore, a structure must be carefully designed such that the proposed geometry will satisfy both equations (2) and (3) with different incident angles, to obtain an angle-insensitive MM absorber. Although a polarization-independent absorber can be easily constructed by arranging horizontally and vertically symmetric unit cells in a periodic array, it is challenging to simultaneously satisfy equations (2) and (3) (and subsequently realize angle insensitivity). Some of the reported structures exhibit stable absorption behaviors up to a certain angle of incidence, and those geometries are generally termed as angularly stable absorber structures. In the following sections, a compressive study of such angularly stable as well as polarization-insensitive MM absorbers are described in detail.

\section{Narrowband metamaterial absorbers}

Narrowband MM absorber structures offer single or multiple absorption peaks, whose resonances are very sharp, and offer limited bandwidths. These structures are primarily used in different types of sensors, imaging instruments, bolometers, and other related applications, where sharp resonances are critical. Such absorbers are typically constructed based on the top metallic pattern resonances, whereas the loss is primarily generated from the dielectric substrates $[22,23]$. The top geometry, made from conductive patches, offers small ohmic losses insufficient for good absorption. Therefore, a lossy dielectric is often preferred in a narrowband absorber structure (unlike other microwave devices) [24].

To achieve narrowband absorption responses, numerous geometries have been explored over the last decade, e.g., square loop, square slot, Jerusalem cross, circular patch, and circular slot [25-30]. However, most of these existing topologies display poor absorption performances under oblique incidence, in particular beyond a certain angle of incidence. Therefore, different strategies have been implemented by various researchers to realize angleindependent MM absorbers. Some of the promising geometries are described in the following sections.

\subsection{Four circular sector-based patch geometry}

Lee et al. have developed a narrowband absorber structure, where the top metallic pattern comprises a center square patch connected with four circular sectors around the patch [31]. The sectors are separated from one another through 


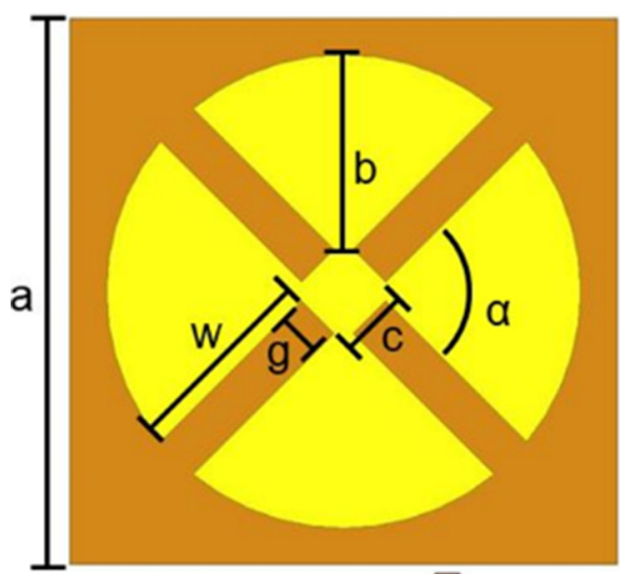

(a)
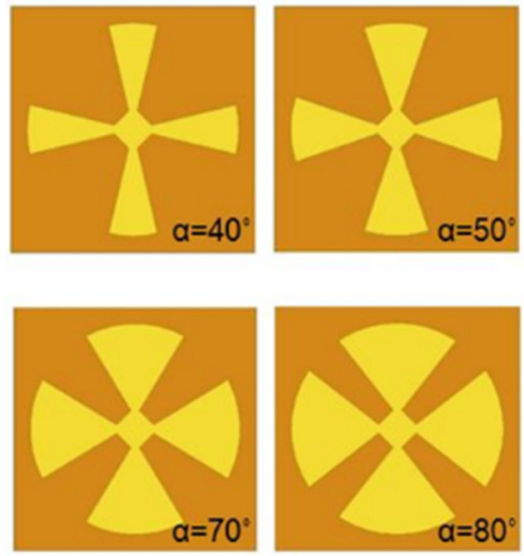

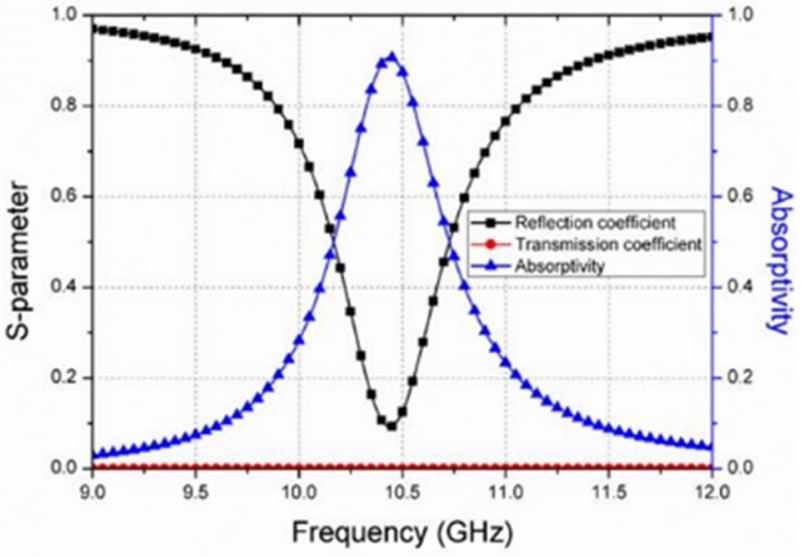

(b)
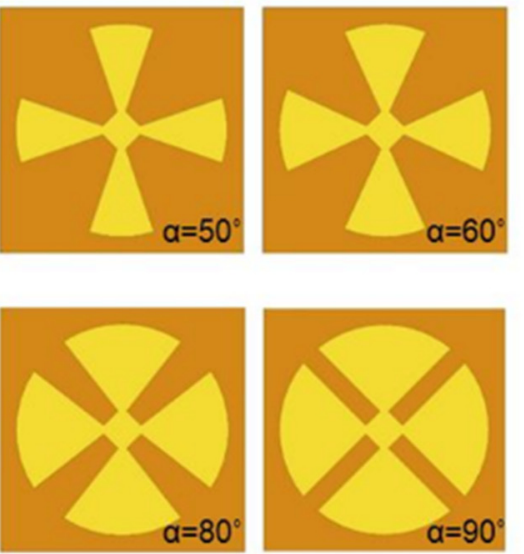

(c)

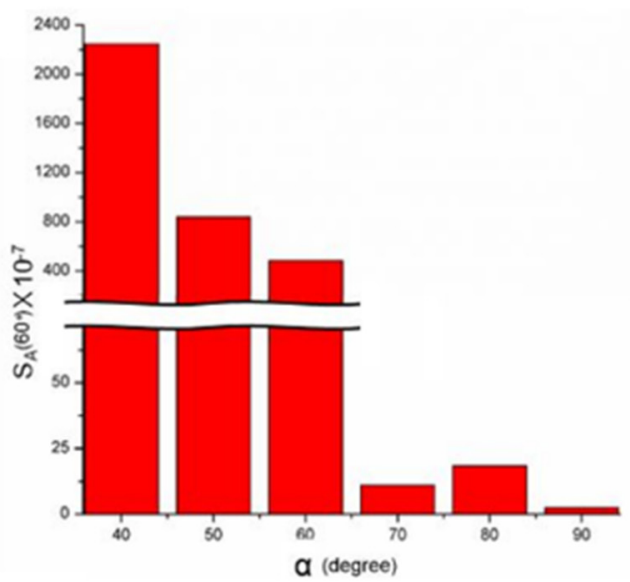

Fig. 1. (a) Proposed unit cell geometry along with its physical dimensions: $a=6 \mathrm{~mm}, b=2.2 \mathrm{~mm}, c=0.8 \mathrm{~mm}, g=0.5 \mathrm{~mm}, w=2.3 \mathrm{~mm}$, $\alpha=90^{\circ}$, and thickness $(t)=0.6 \mathrm{~mm}$. (b) Simulated reflection coefficient, transmission coefficient, and absorptivity of the optimized structure. (c) Angle-sensitivity $\left(S_{A}\right)$ of the topology for different values of the inner angle $(\alpha)$ and corresponding geometries [31].

symmetric slots to realize the capacitive effect. The overall pattern was printed on a substrate, whose rear side was completely metal laminated. FR4 (relative permittivity $\left(\varepsilon_{r}\right)$ of 4.4 and dielectric loss tangent $(\tan \delta)$ of 0.02$)$ was used as the dielectric in the structure. The top and bottom metal patches were made of copper having a conductivity $(\sigma)$ of $5.8 \times 10^{7} \mathrm{~S} / \mathrm{m}$ and a thickness of $0.035 \mathrm{~mm}$. The physical dimensions along with the unit cell geometry of the proposed absorber are presented in Figure 1a.

The top metallic patch exerts an equivalent inductance $(L)$ and capacitance $(C)$ to the geometry, whereas the grounded dielectric, acting as a short-circuited transmission line, provides an imaginary impedance. An equivalent resistance $(R)$ is obtained owing to the finite conductivity of the metal. Therefore, the input impedance of the structure can be expressed as $[32,33]$

$$
\begin{aligned}
& Z_{\text {in }}=\{R+j \omega L+1 / j \omega C\} \| Z_{d}, \\
& Z_{d}=j \sqrt{\mu_{r} \mu_{0} / \varepsilon_{r} \varepsilon_{0}} \tan (k t) .
\end{aligned}
$$

Here, $\varepsilon_{r}$ and $\mu_{r}$ are the real parts of the relative permittivity and permeability of the dielectric, respectively. $k=k_{0} \sqrt{\varepsilon_{r} \mu_{r}}$ is the wavenumber of the incident EM wave. Therefore, by controlling the geometric parameters of the topology, the input impedance of the structure $\left(Z_{\text {in }}\right)$ can be perfectly matched to the free space impedance $\left(Z_{0}\right)$ at a particular frequency. This results in minimal reflection coefficient, whereas the transmission coefficient is also zero (due to the presence of ground plane). Then, the incident EM wave gets completely absorbed in the geometry through the ohmic loss (in the top metallic layer) and the dielectric loss (in the intermediate substrate).

Because the primary focus is to design an angularly stable MM absorber, other criteria must be satisfied along with the impedance matching condition [34]. An angle-insensitive absorber structure not only retains the absorptivity magnitude with higher incident angles, the absorption frequency also remains constant. Although some of the earlier geometries (e.g., Jerusalem cross) result in high absorption under oblique incidence, their 
absorption frequencies become deviated at higher incident angles [31]. Thus, the sole purpose of designing the angleinsensitive absorber has become futile. In the proposed geometry, both the absorptivity and absorption frequency have been maintained relatively constant to obtain an angularly stable absorber structure.

While investigating the proposed structure for various incident angles, it is found that the deviation in the absorption response under oblique incidence can be significantly reduced by optimizing some of the physical dimensions of the geometry. A theoretical term, i.e., angle sensitivity $\left(S_{A}\right)$, has been defined as

$$
S_{A}(\theta)=\frac{\left(f_{\theta}-f_{0}\right) \times\left[A\left(\theta, f_{\theta}\right)-A\left(0, f_{0}\right)\right]}{(\theta-0)^{2}},
$$

where $f_{\theta}$ and $f_{0}$ are the resonant frequencies at the angles of incidence $\theta^{\circ}$ and $0^{\circ}$ (i.e., normal incidence), respectively. $A\left(\theta, f_{\theta}\right)$ is the absorption magnitude corresponding to an absorption frequency $f_{\theta}$ at $\theta^{\circ}$ incident angle. $A\left(0, f_{0}\right)$ is the absorptivity under normal incidence, where the absorption occurs at $f_{0}$ frequency. As shown in equation (6), the smaller the value of angle sensitivity $\left(S_{A}\right)$, the more angle insensitive the structure.

Figure 1c shows a histogram plotting of $S_{A}$ for different values of the parameter $\alpha$, where $\alpha$ is the inner angle at each of the circular sectors. As $S_{A}$ exhibits the lowest value corresponding to $\alpha=90^{\circ}$, the structure is optimized for this set of dimensions, and the simulated scattering coefficients are presented in Figure 1b. The unit cell geometry of the proposed structure is simulated in a finite-elementmethod-based EM solver ANSYS high-frequency structure simulator (HFSS) software, using periodic boundary conditions. Floquet port excitations are utilized in the simulation setup to mimic the infinite periodic structure into a unit cell configuration. The transmission coefficient is zero owing to metal backing, whereas a narrowband reflection dip is observed at $10.44 \mathrm{GHz}$. This corresponds to a near-unity absorption at $10.44 \mathrm{GHz}$ having an absorptivity of $91 \%$, as depicted in Figure $1 \mathrm{~b}$.

The response above has been analyzed under normal incidence. The structure must be studied for different polarizations as well as incident angles to confirm its polarization- and angle-insensitive characteristics. During simulation, the structure exhibits almost similar absorption responses for all the polarization angles under normal incidence, as observed in Figure 2a. In the simulation setup, the incident wave propagation direction remains constant, whereas the incident electric and magnetic field vector directions are varied with the angle of polarization $(\phi)$. Physically, an incident electric field vector of any arbitrary polarization can be divided into two components: vertical and horizontal. Owing to the fourfold symmetric geometry, the design exhibits identical behaviors for both modes, thereby providing polarizationindependent characteristic.

The proposed geometry has also been studied under oblique incidence. Two different cases may occur under oblique incidence: in one condition, the incident electric field direction remains constant, whereas the magnetic field and wave propagation directions are rotated with the incident angle $(\theta)$. This is known as perpendicular polarization or transverse electric (TE) polarization. The modified reflection coefficient term during TE polarization is expressed in equation (2). On the contrary, the incident magnetic field direction remains the same, and the electric field and wave propagation vector directions are varied with the incident angle in the other case. This phenomenon is known as parallel polarization or transverse magnetic (TM) polarization. Equation (3) shows the reflection coefficient formula corresponding to this mode. During simulation, phase delay between the master and slave boundary is varied to change the incident angle of the EM wave, whereas the selection of the polarization (TE/TM) can be regulated by the floquet port excitation. Figures $2 \mathrm{~b}$ and $2 \mathrm{c}$ display the simulated absorption responses of the proposed structure under TE and TM polarizations, respectively. As observed, the geometry exhibits an absorptivity higher than $90 \%$, and the variation in the absorption frequency becomes less than $0.96 \%$ for incident angles up to $70^{\circ}$. Therefore, the design can be considered as an angularly stable MM absorber, as claimed by the authors.

To demonstrate the proposed absorber, a prototype was fabricated on a commercial FR4 substrate using printed circuit board (PCB) technology and measured in the anechoic chamber using the free space technique. The sample exhibited good agreement between the measured and simulated responses under normal as well as oblique incidences.

\subsection{Four circular sector-based slot geometry}

Nguyen et al. have later modified the topology above and proposed a narrowband absorber based on its complement geometry (with some modifications in the physical dimensions) [35]. Figure 3a illustrates the unit cell configuration, where the top metallic pattern is based on a slot geometry, and the substrate is backed by a ground plane. The geometry, owing to inductive and capacitive effects, results in a narrowband absorption at $10.05 \mathrm{GHz}$ with $91.48 \%$ absorptivity. The structure has also been studied for different values of the internal angle $(\alpha)$ and revealed that the geometry achieves the least angle sensitivity $\left(S_{A}\right)$ performance at $\alpha=85^{\circ}$. Figure $3 \mathrm{~b}$ shows that the simulated absorptivity of the optimized structure under TE polarization exceeds $90 \%$ absorptivity in all the cases until $\theta=70^{\circ}$. However, the proposed design suffers from two disadvantages: (i) an additional absorption peak is observed at approximately $11 \mathrm{GHz}$ during larger incident angles owing to the higher order resonance; (ii) the absorption frequency shift is considerably large during TM polarization (not shown here). In an aperture-based geometry, effective inductance and capacitance occur in parallel combination, and their contributions significantly get deviated with the incident electric field [36]. This subsequently shifts the absorption frequency under TMpolarized incident EM wave. Therefore, it can be concluded that the previous patch-based geometry provides better angular stability response compared to this slot topology. Hence, the subsequent absorber structures discussed herein are based on the patch configuration only. 


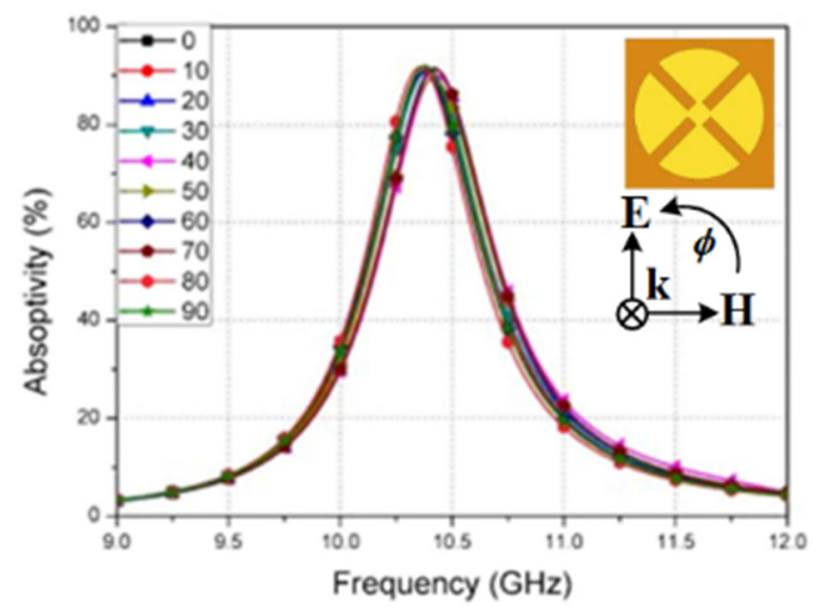

(a)

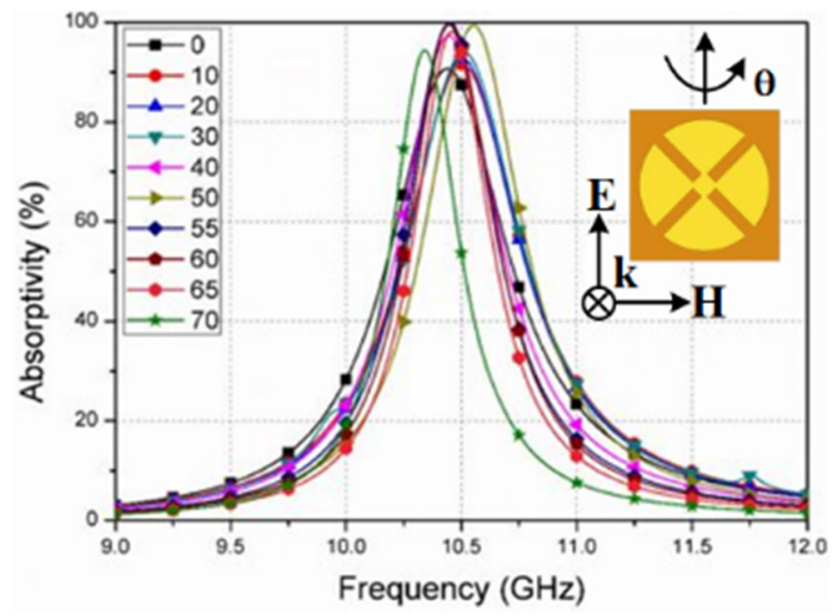

(b)

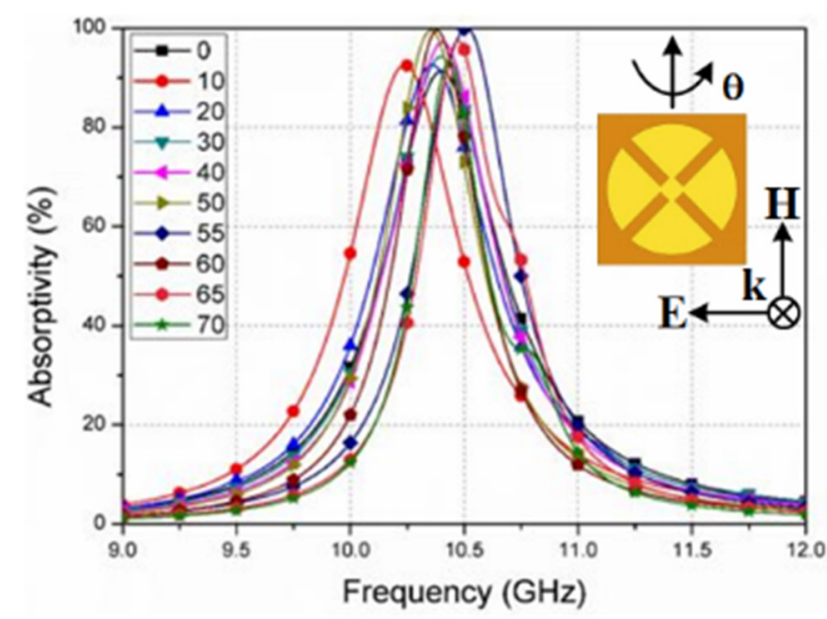

(c)

Fig. 2. Simulated absorptivity of the proposed absorber (a) for different polarization angle under normal incidence, and for different incident angles under (b) TE polarization, and (c) TM polarization [31].

\subsection{Eight circular sector-based patch geometry}

From the earlier observations, the patch geometry was shown to provide a better angularly stable response. Therefore, the concept has been further extended by increasing the number of patches in the circular sector topology. Nguyen and Lim have designed another narrowband absorber structure, whose unit cell geometry comprises eight circular sector patterns on the top of a grounded dielectric [37]. The properties of the substrate and metal are similar, as mentioned in the previous geometries. The proposed absorber design is presented in Figures $4 \mathrm{a}$ and $4 \mathrm{~b}$.

Owing to the larger number of circular sectors, the topology exhibits an improved angular response compared to the previous two absorbers [38]. Here, the absorption frequency is observed at $9.26 \mathrm{GHz}$ with $96 \%$ absorptivity at normal incidence. The structure, while simulated under oblique incidence, displays an absorptivity higher than
$90 \%$ until $70^{\circ}$ incident angle, and the absorption frequency variation is limited to within $0.7 \%$, for both TE and TM polarizations. Figures $4 \mathrm{~b}$ and $4 \mathrm{c}$ depict the simulated responses of the proposed structure for different incident angles. Our experimental validation has also confirmed the proposed absorber concept.

\subsection{Via-array-based geometry}

In addition to the circular sector-based geometry, other techniques can be used to realize angle-insensitive MM absorbers. Lim et al. have presented a via-array-based structure that exhibits an improved angular response compared to its counterpart [39]. The proposed design consists of a split ring cross-resonator arranged periodically on a grounded dielectric substrate. The properties of the dielectric and the metal are similar, as previously reported. Figure 5 illustrates the proposed structure along with its physical dimensions. 


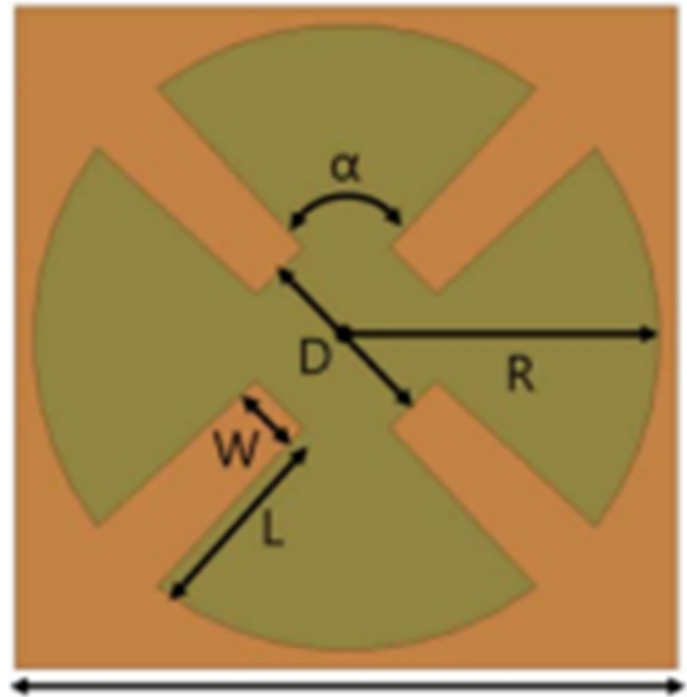

P

(a)

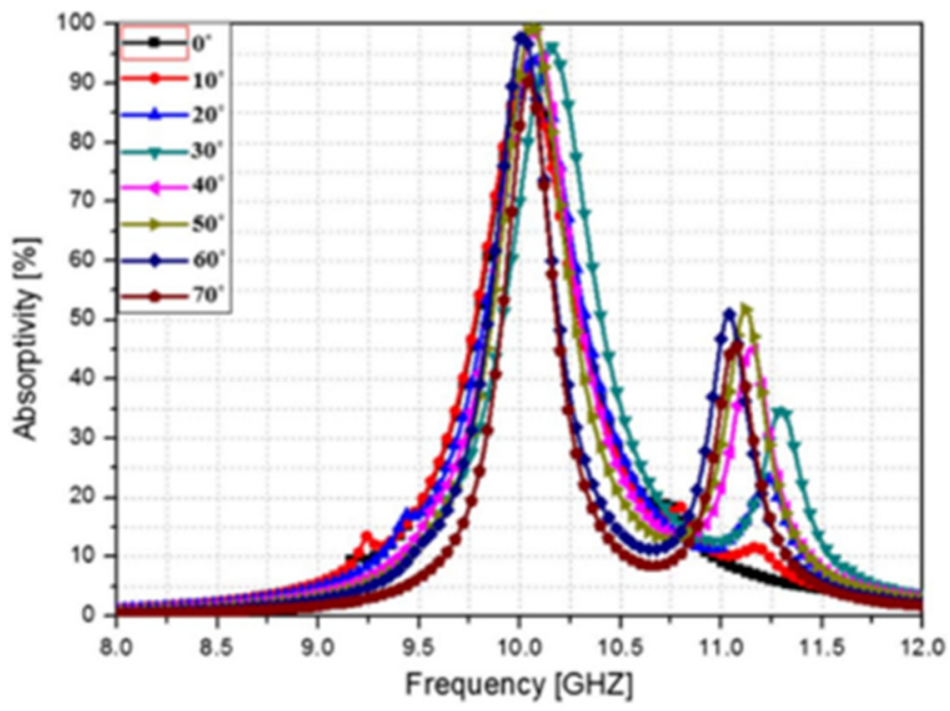

(b)

Fig. 3. (a) Proposed unit cell geometry along with its physical dimensions: $P=9.1 \mathrm{~mm}, R=4.3 \mathrm{~mm}, W=0.9 \mathrm{~mm}, D=2.6 \mathrm{~mm}$, $L=2.96 \mathrm{~mm}$, thickness $=0.8 \mathrm{~mm}$, and $\alpha=85^{\circ}$. (b) Simulated absorptivity of the proposed structure for different incident angles under TE mode [35].

The geometry, with and without metallic vias, exhibits narrowband absorption at 11.31 and $11.35 \mathrm{GHz}$, respectively, at $0^{\circ}$ incident angle. Thus, no significant difference is observed with the inclusion of the via array, particularly at normal incidence. However, when the incident angle is gradually increased, both the absorptivity and absorption frequency deviate from that of the normal incidence response for the via-less structure, as observed in Figure 6a. On the contrary, the absorption frequency remains almost constant under oblique incidence after the inclusion of the via array, as depicted in Figure 6b. As the incidence angle gets wider, the coupling between adjacent split ring crossresonators becomes higher. The angle insensitivity is achieved by reduction of coupling level due to the proposed via array. The proposed geometry was also fabricated using PCB technique, measured under normal and oblique incidences, and was found to exhibit good agreement between the simulated and measured responses.

\subsection{Multilayer geometry}

Multilayer technology can also be used to produce MM absorbers [40,41]. Yoo et al. have proposed an MM absorber based on a multilayer geometry to reduce the electrical size of the unit cell, as well as to improve the angular response of the structure [42]. Figure 7 depicts the unit cell configuration of the structure, which consists of three layers: a conductive ring in the top layer, a split ring cross-resonator in the middle layer, and inductive meander lines in the bottom layer. Metallic vias were used to connect the middle and bottom layer patterns, which lower the absorption frequency owing to the increase in the capacitance value. The overall geometry is backed by a metal ground to eliminate transmissions through the structure. A significant improvement in the angular response of the geometry, owing to this metal-insulator-metal capacitance, has also been experimentally confirmed, as shown in Figures $7 \mathrm{c}$ and $7 \mathrm{~d}$. The structure resulted in an absorptivity higher than $90 \%$ until $40^{\circ}$ incident angle for TE polarization, whereas almost perfect absorptivity (above 95\%) was observed until $60^{\circ}$ during TM polarization.

\section{Broadband metamaterial absorbers}

Because MM absorbers are produced from the resonance mechanism, the absorption bandwidths are typically narrow. These narrowband absorbers, although suitable for limited applications, cannot be utilized in other operations, e.g., stealth technology [43] and wideband shielding [44]. Therefore, broadband absorbers covering several microwave bands $(\mathrm{C}, \mathrm{X})$ are in great demand. Before realizing broadband absorbers, researchers have attempted to increase the absorption bandwidths originating primarily from the existing narrowband absorbers [45-48]. This produces a number of bandwidth-enhanced geometries, whose bandwidths are considerably larger than those of the narrowband absorbers, but smaller than those of the broadband structures.

\subsection{Bandwidth-enhanced absorber using hybrid geometry}

One of the conventional techniques to realize a bandwidthenhanced absorber is to use a hybrid unit cell geometry $[49,50]$. Two different sets of resonating structures (having different absorption frequencies) are embedded in a single unit cell that exhibit two absorption peaks. By properly 

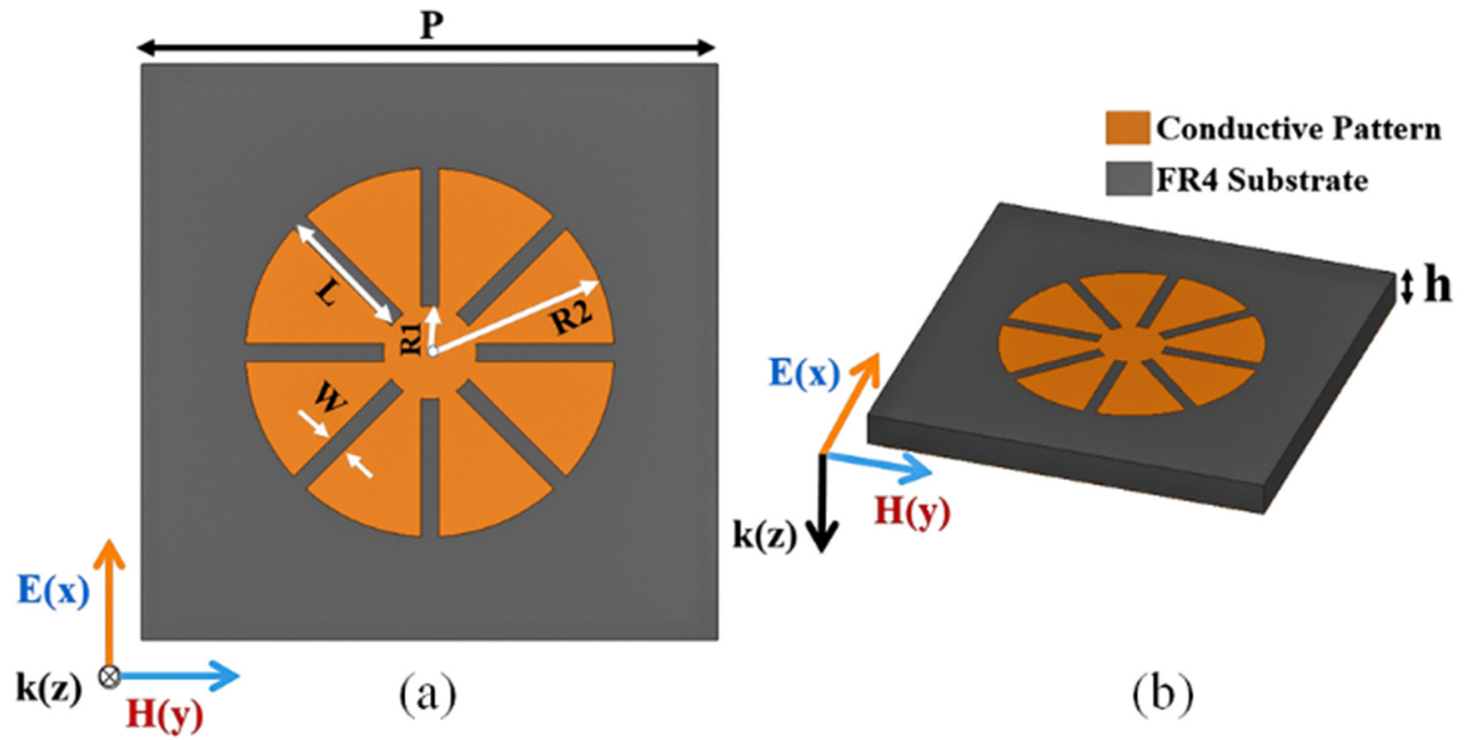

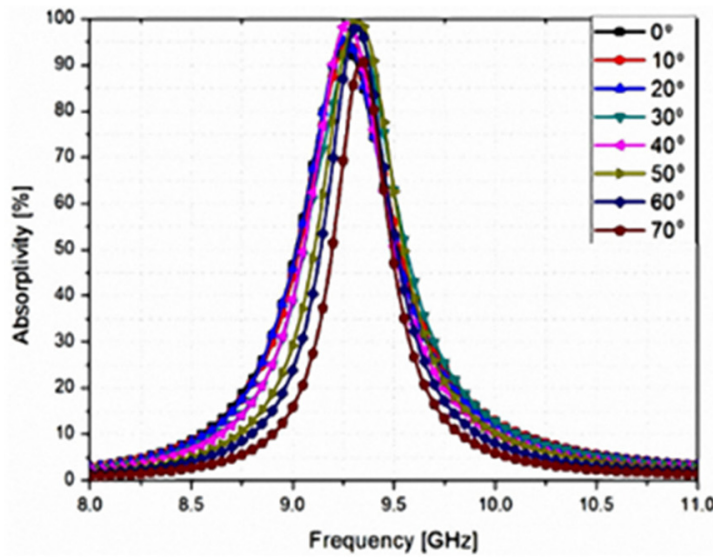

(c)

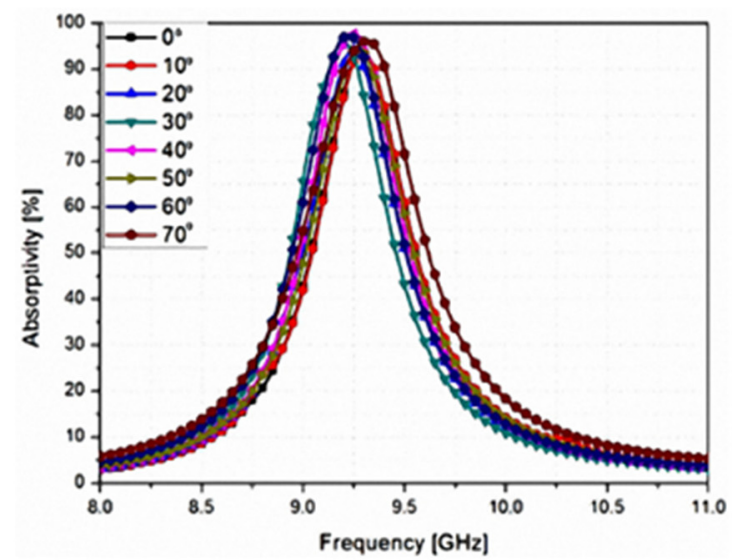

(d)

Fig. 4. Proposed unit cell geometry along with its physical dimensions: $P=10 \mathrm{~mm}, R_{1}=0.81 \mathrm{~mm}, R_{2}=3.2 \mathrm{~mm}, L=2.4 \mathrm{~mm}$, $W=0.3 \mathrm{~mm}$, and thickness $=0.8 \mathrm{~mm}$. (a) Top view. (b) Perspective view. Simulated absorptivity of the proposed absorber for different incident angles under (b) TE mode and (c) TM mode [37].

optimizing the design parameters of the structure, these two frequencies can be brought closer to realize a bandwidth-enhanced absorption.

A few bandwidth-enhanced MM absorbers have been presented based on the technique above; however, most of these structures exhibit poor angular responses under oblique incidence. Because the circular sector-based geometry has resulted in an improved absorption response at higher incident angles, Nguyen and Lim have implemented the concept in achieving a bandwidth-enhanced absorber with angle insensitivity [51]. The proposed geometry comprises a periodic arrangement of four circular sector metallic patches imprinted on a grounded dielectric substrate, as shown in Figure 8a. In the unit cell, the first and fourth patterns (marked "A") are identical, whereas the second and third patches (marked "B") are similar. These two variations in the circular sector geometries are different from each other by a few geometric dimensions. Owing to the small difference in the parameters, these two sets of resonating structures give rise to two distinct absorption peaks that are close to each other. By properly optimizing the dimensions, these peaks have been brought together, and resulted in a bandwidth-enhanced absorber. The absorption responses of the individual metallic patches ("A" and "B") along with the combined response are illustrated in Figure 8b. The "A" and "B" patches exhibit near-unity absorption at 10.38 and $10.55 \mathrm{GHz}$, respectively, whereas the hybrid unit cell results in an enhanced absorption bandwidth from 10.25 to $10.65 \mathrm{GHz}$. Thus, the fractional bandwidth increases to $7.66 \%$ from $1.94 \%$, as obtained from the single peak geometry [31].

As the unit cell topology is fourfold symmetric, the structure provides a constant absorption bandwidth and frequency for all the polarization angles. The fabricated prototype was also measured under different polarization angles, and the polarization-insensitive characteristics were experimentally demonstrated. Figures $8 \mathrm{c}$ and $8 \mathrm{~d}$ depict the simulated absorption behaviors of the geometry at different incident angles under TE and TM modes, respectively, and it is found that the absorption magnitude exceeds $91 \%$ at 


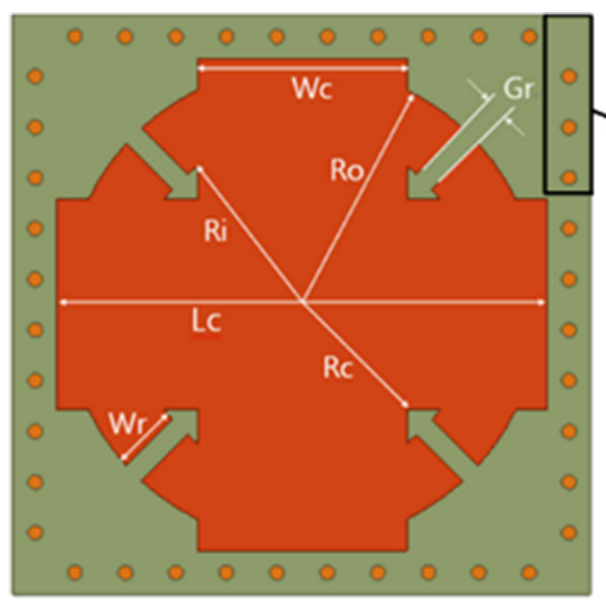

(a)

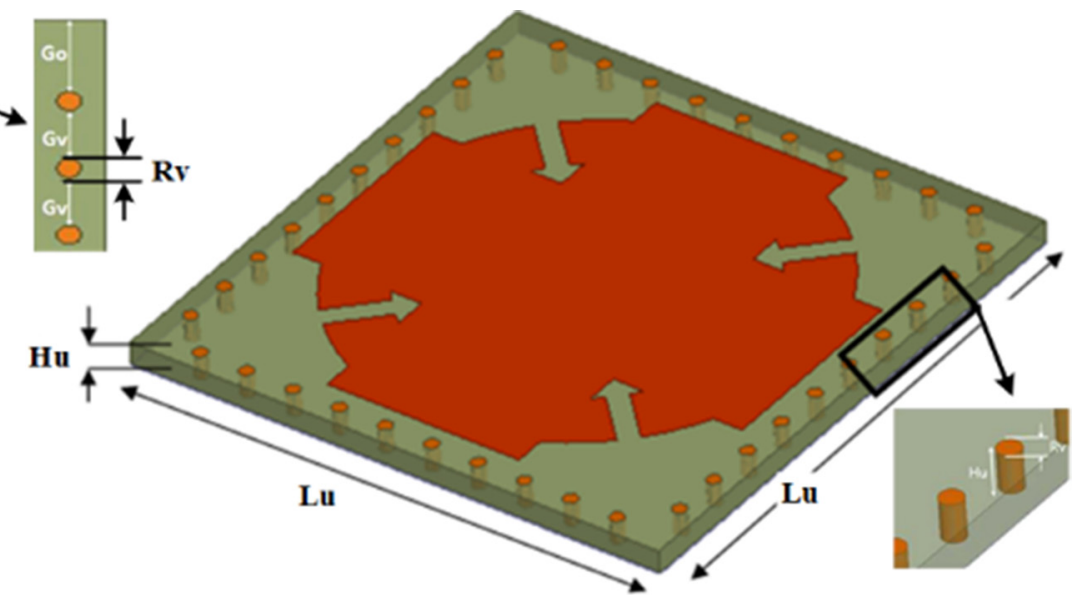

(b)

Fig. 5. Unit cell geometry of the proposed absorber structure: (a) top view and (b) perspective view. The optimized physical dimensions are as follows: $\mathrm{Lu}=8 \mathrm{~mm}, \mathrm{Lc}=6.8 \mathrm{~mm}, \mathrm{Ro}=3.3 \mathrm{~mm}, \mathrm{Ri}=2.4 \mathrm{~mm}, \mathrm{Rc}=2.05 \mathrm{~mm}, \mathrm{Wr}=0.9 \mathrm{~mm}, \mathrm{Wc}=2.9 \mathrm{~mm}$, $\mathrm{Gc}=0.3 \mathrm{~mm}, \mathrm{Rv}=0.2 \mathrm{~mm}, \mathrm{Go}=0.75 \mathrm{~mm}, \mathrm{Gv}=0.5 \mathrm{~mm}$, and $\mathrm{Hu}=0.35 \mathrm{~mm}$ [39].

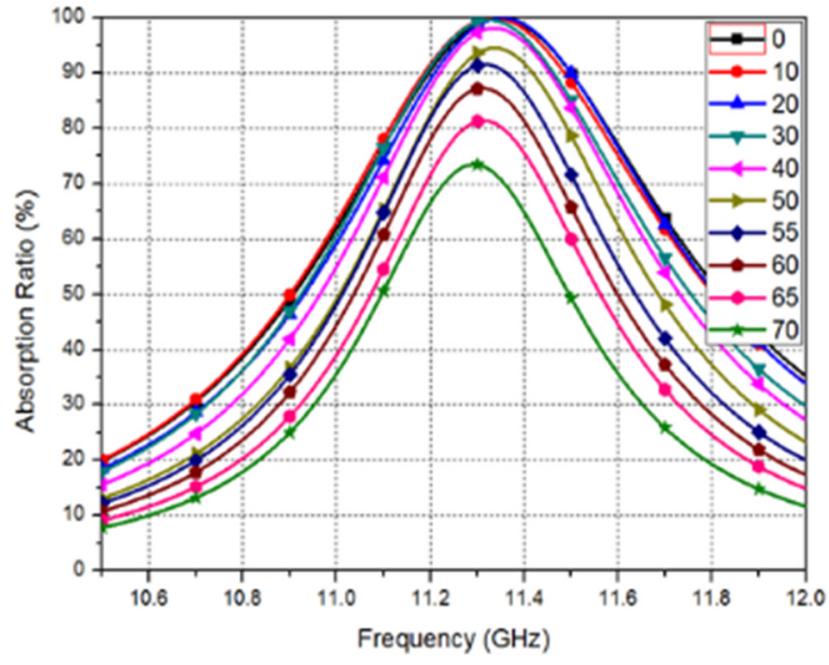

(a)

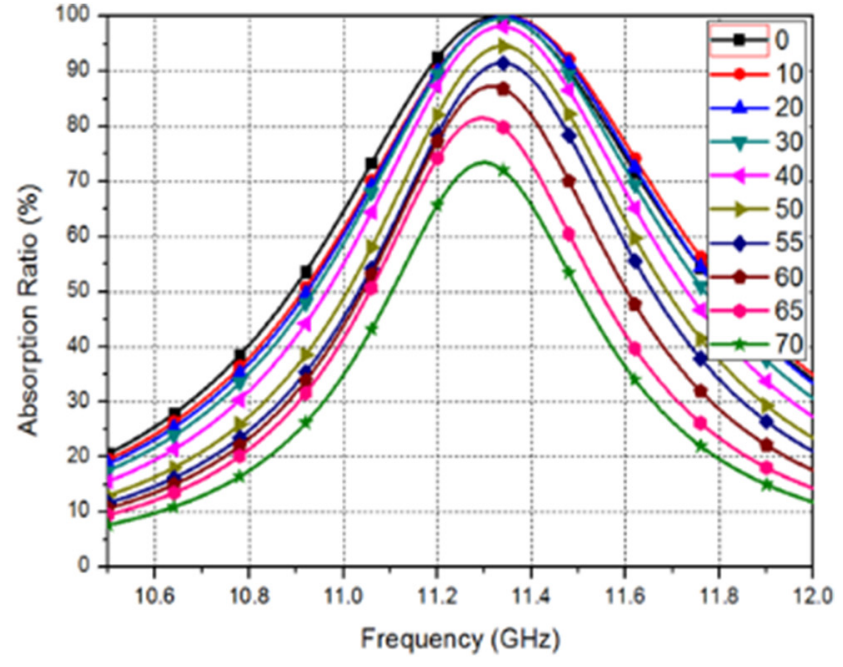

(b)

Fig. 6. Simulated absorptivities of the proposed structure under TE polarization: (a) without via array and (b) with via array [39].

approximately $10.41 \mathrm{GHz}$ at an angle of incidence up to $70^{\circ}$ in both the polarizations. The measured responses display an absorptivity above $90 \%$ at the center frequency until $70^{\circ}$ incident angle; thereafter, the absorption responses gradually reduce. The proposed structure has also been compared with earlier reported bandwidth-enhanced absorbers, and was found to exhibit larger absorption bandwidths, in particular at higher incident angles $\left(60^{\circ}\right.$ and $\left.70^{\circ}\right)$ for $\mathrm{TE}$ and TM polarizations.

\subsection{Broadband absorber using four circular sector geometry}

Although the technique above exhibits a bandwidthenhanced MM absorber, the absorption bandwidth is limited to a few hundred megahertz only. However, most of the practical applications demand an absorption bandwidth on the order of a few gigahertz, along with an absorptivity level above $90 \%$. Therefore, new absorption 

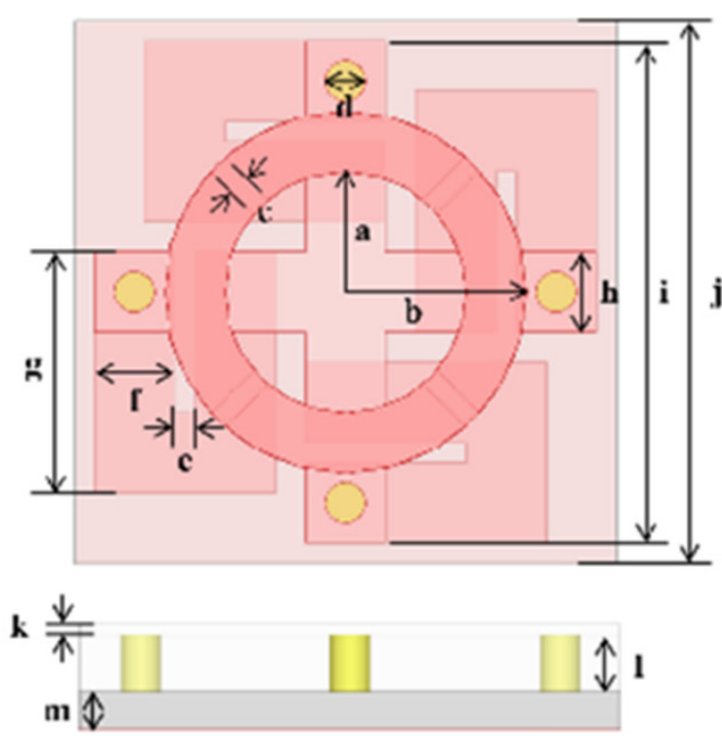

(a)

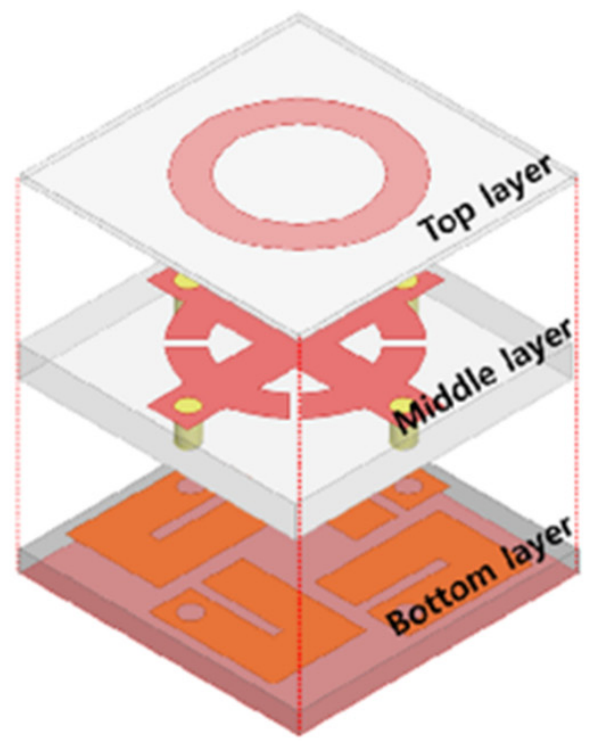

(b)

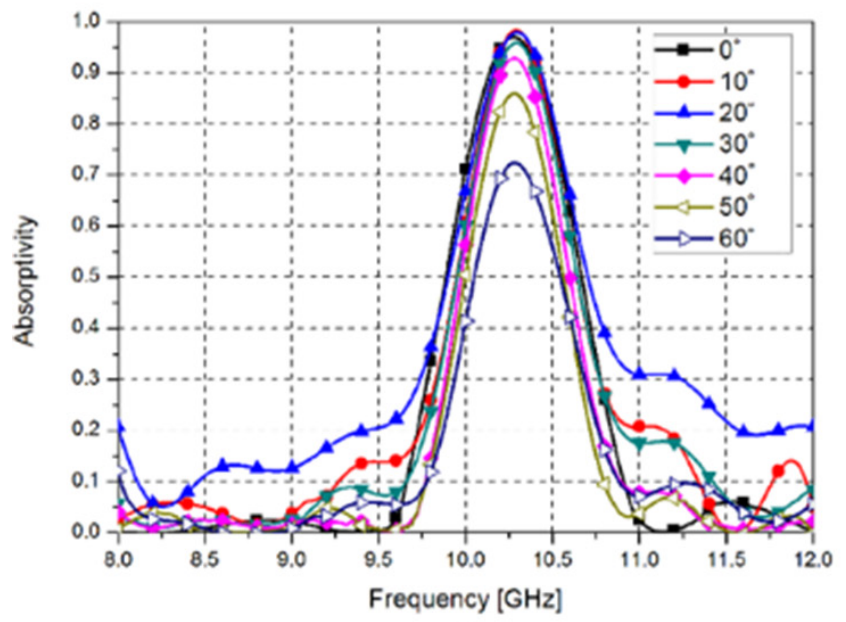

(c)

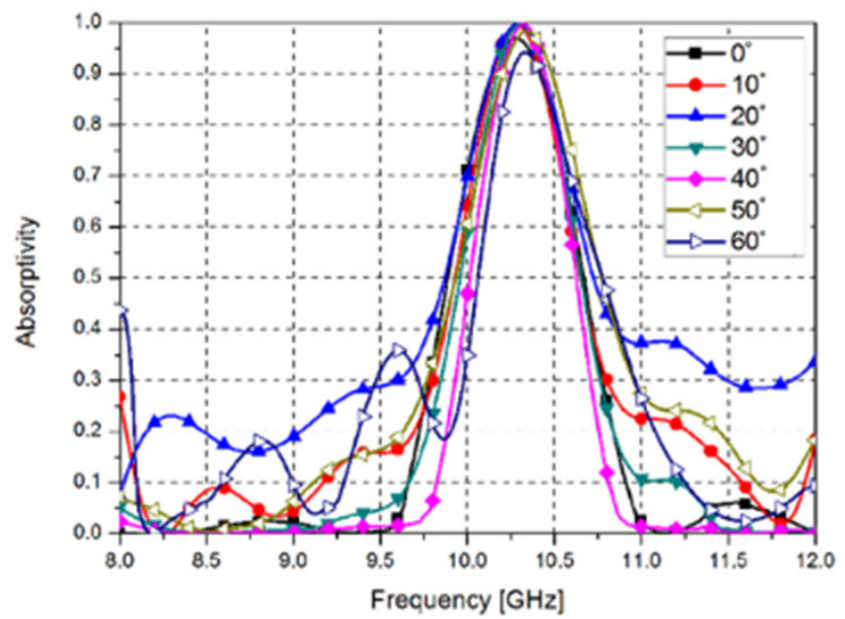

(d)

Fig. 7. Unit cell geometry of the proposed absorber: (a) top view and (b) perspective view. The geometric dimensions are $a=0.6 \mathrm{~mm}$, $b=0.9 \mathrm{~mm}, c=0.1 \mathrm{~mm}, d=0.2 \mathrm{~mm}, e=0.1 \mathrm{~mm}, f=0.4 \mathrm{~mm}, g=1.2 \mathrm{~mm}, h=0.4 \mathrm{~mm}, i=2.5 \mathrm{~mm}, j=2.7 \mathrm{~mm}, k=0.06 \mathrm{~mm}, l=0.3 \mathrm{~mm}$, and $m=0.2 \mathrm{~mm}$. Measured absorptivity of the proposed absorber at different incident angles under (c) TE polarization (d) TM polarization [42].

mechanisms are to be implemented for realizing such wideband absorbers.

Nguyen and Lim have therefore incorporated the circuit analog (CA) concept in the circular sector geometry to increase the absorption bandwidth [52]. A CA-based absorber is an effective solution to resolve the narrow bandwidth problem, where broadband absorption is obtained by appropriately depositing resistive and conductive patterns on a dielectric substrate [53]. This periodic resistive-conductive pattern of the $\mathrm{CA}$ absorber can be realized by mounting lumped resistors across the gaps present in the metallic patterns printed on the dielectric substrates [54-57].
The proposed design is composed of four circular patch sectors connected at the center through a square patch, and the overall geometry is patterned on a grounded dielectric substrate in a periodic array. The properties of the dielectric and metal are similar, as mentioned in the previous geometries. To increase the absorption bandwidth, four lumped resistors are symmetrically mounted across the slots between the circular sectors, as illustrated in Figure 9a. The lumped resistors, owing to a high ohmic loss, absorb the incident EM wave over a large frequency range and exhibit broadband absorption (above 90\%) from 7.1 to 13.5 GHz under normal incidence. 


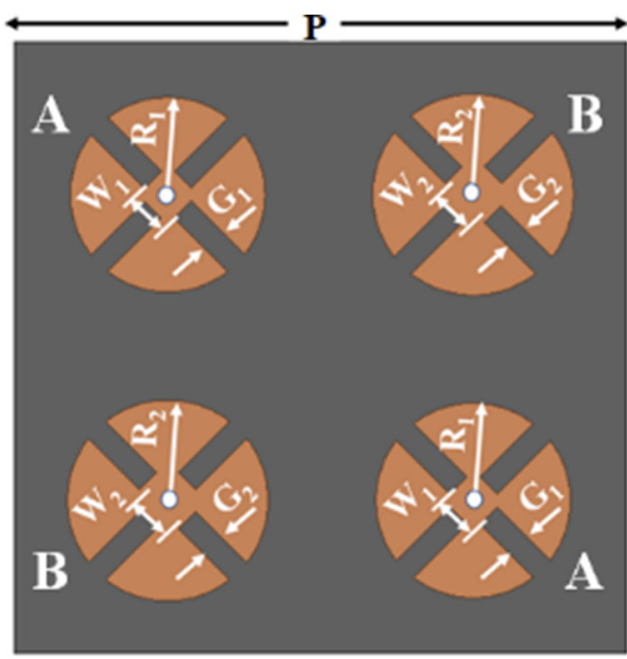

(a)

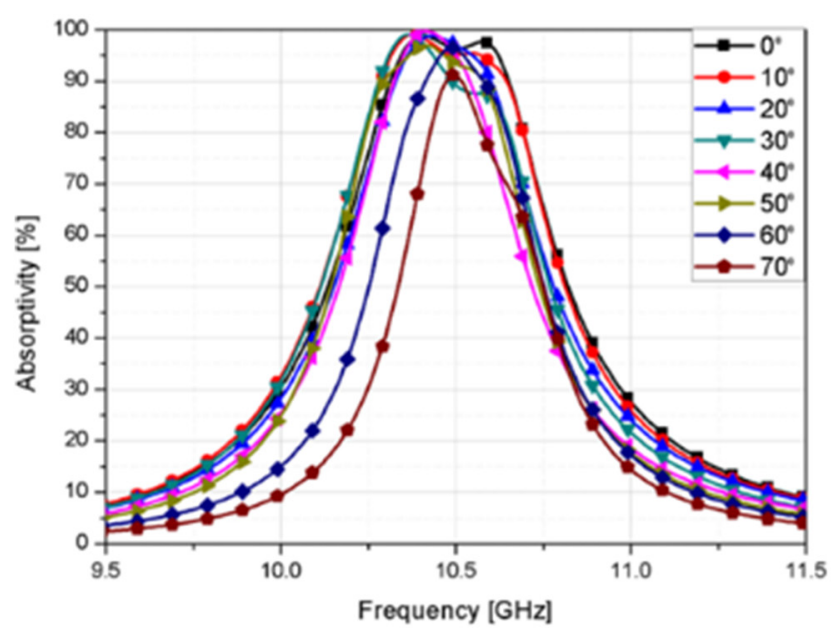

(c)

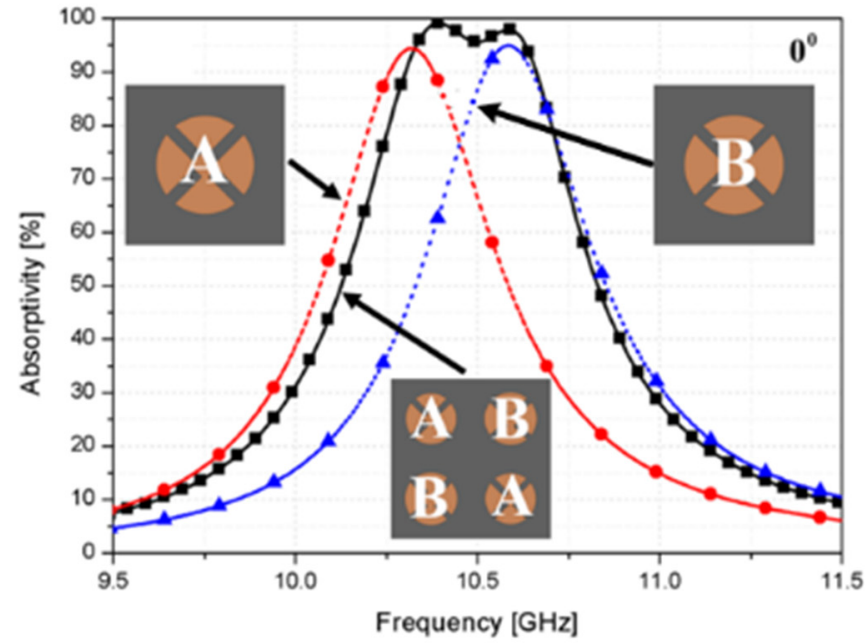

(b)

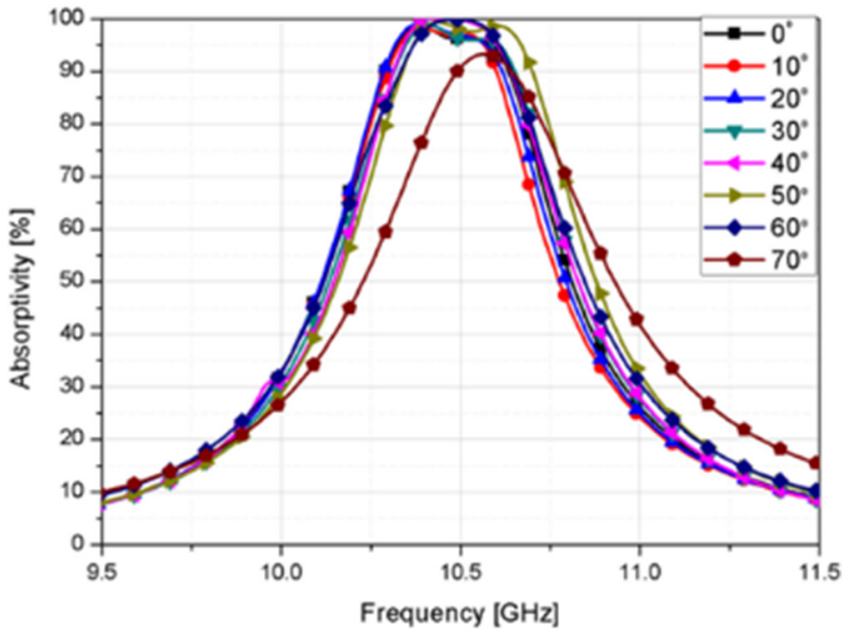

(d)

Fig. 8. (a) Top view of the proposed bandwidth-enhanced MM absorber. The geometric dimensions are as follows: $P=17.6 \mathrm{~mm}$, $R_{1}=2.8 \mathrm{~mm}, \quad R_{2}=2.89 \mathrm{~mm}, W_{1}=1.22 \mathrm{~mm}, W_{2}=1.6 \mathrm{~mm}, G_{1}=0.7 \mathrm{~mm}, \quad G_{2}=0.7 \mathrm{~mm}$, and thickness $=0.8 \mathrm{~mm}$. (b) Simulated absorptivity of the proposed structure along with its individual counterparts under normal incidence. Simulated absorptivity of the proposed structure at different incident angles under (c) TE polarization and (d) TM polarization [51].

Because the geometry is fourfold symmetric and the lumped components are mounted symmetrically, the structure provides identical absorption responses for all the polarization angles. Therefore, the topology can be considered as polarization independent. The proposed structure has also been studied under oblique incidence, as depicted in Figures 9c and 9d. In the TE mode, the absorption magnitude slowly reduces with higher incident angles. Nevertheless, the broadband absorptivity of above $90 \%$ is still achieved between 7 and $12.2 \mathrm{GHz}$ up to $50^{\circ}$ incident angle.

However, the absorption characteristics change differently under TM polarization. Instead of a gradual reduction in the absorption bandwidth, a sharp decrease occurred in the absorptivity at approximately $10 \mathrm{GHz}$ with higher incident angles, and this effectively divides the absorption bandwidth into two spectra. This results in a reduced absorption from 7.1 to $9.4 \mathrm{GHz}$ at $50^{\circ}$ incident angle. Therefore, the proposed structure, although exhibiting polarization-insensitive broadband absorption under normal incidence, cannot provide good angularly stable characteristics, in particular during TM polarization.

\subsection{Broadband absorber using eight circular sector geometry}

The disadvantage (of having poor absorption performance under TM mode) in the previous structure has been improved in the subsequent geometry, as presented by Nguyen and Lim [58]. The proposed geometry consists of an eight-resistive arm arranged circularly on a grounded dielectric substrate. Lumped resistors of $100 \Omega$ are 


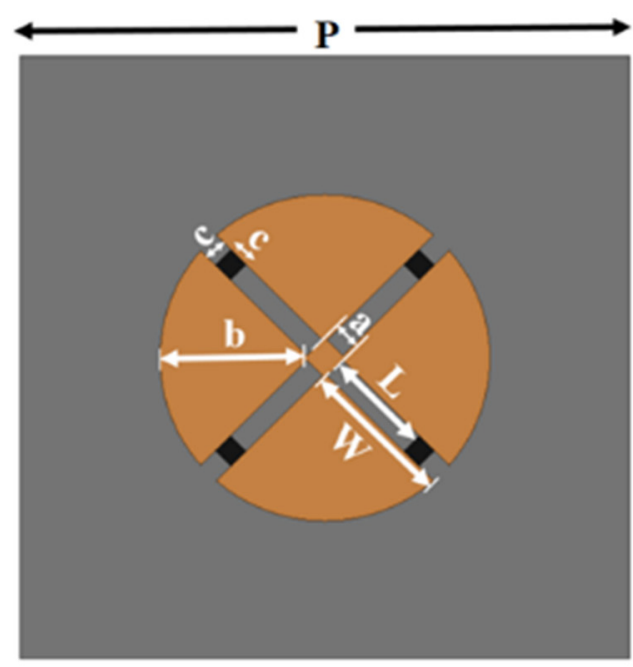

(a)

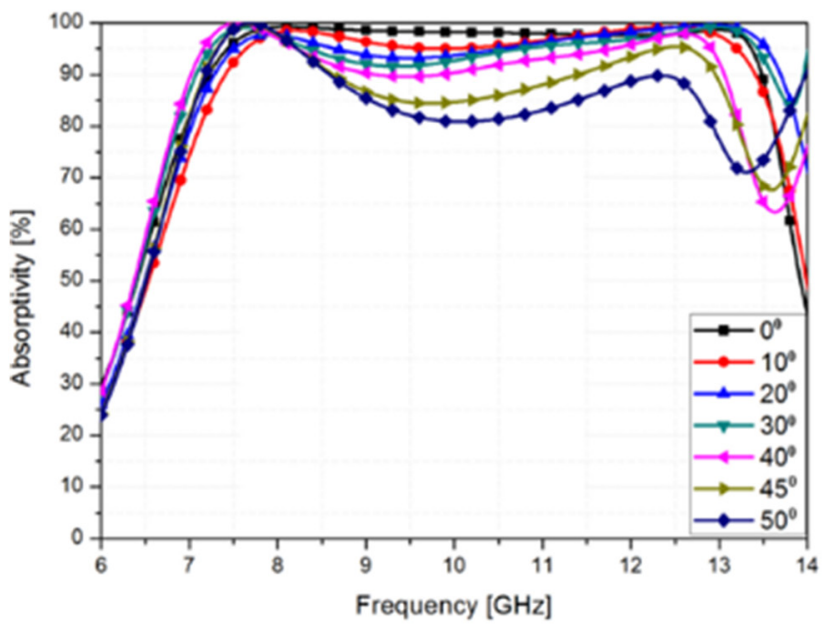

(c)

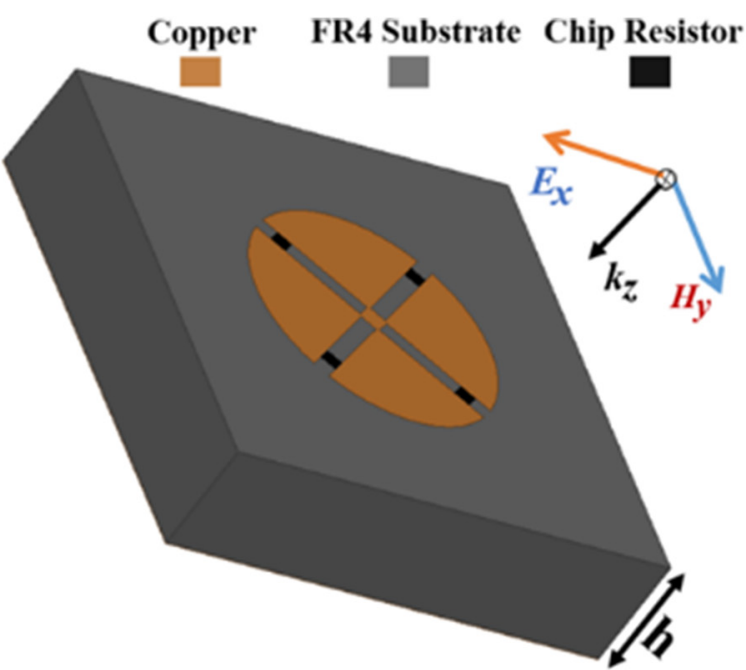

(b)

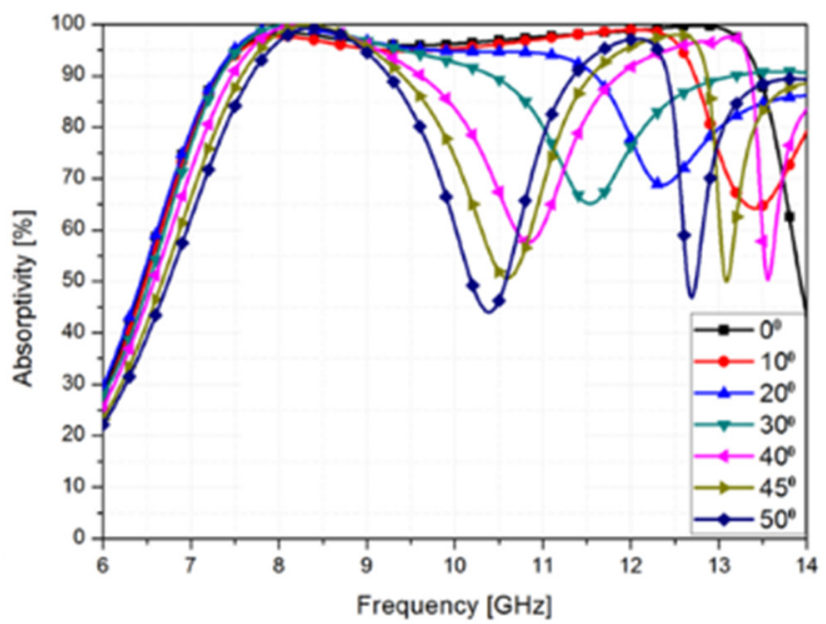

(d)

Fig. 9. Proposed broadband absorber with four circular sector geometry: (a) top view and (b) side view. The optimized geometric dimensions are the following: $P=14 \mathrm{~mm}, W=3.45 \mathrm{~mm}, L=2.485 \mathrm{~mm}, a=0.65 \mathrm{~mm}, b=3.4 \mathrm{~mm}, c=0.5 \mathrm{~mm}, h=3.4 \mathrm{~mm}$, and $R=150 \Omega$. Simulated absorptivity of the proposed structure at different incident angles under (c) TE polarization and (d) TM polarization [52].

mounted across the gaps in each of the arms to realize wideband absorption. With the increase in the number of arms, the topology becomes eightfold symmetric, unlike the fourfold symmetry in the previous geometry. This results in an improved angular response of the structure. Figures 10a and $10 \mathrm{~b}$ illustrate the top view and perspective view, respectively, of the unit cell geometry of the proposed absorber.

When the geometry without the lumped resistors is studied, two narrowband absorption frequencies are obtained at 8.89 and $13.20 \mathrm{GHz}$, as observed in Figure 10c. These sharp resonances are generated owing to the similar absorption mechanisms described in the previous section. However, with the inclusion of the lumped components, the incident EM wave gets absorbed over a wide frequency range and exhibits an absorptivity greater than $90 \%$ in the range of 8.2-13.4 GHz. This corresponds to a fractional bandwidth of $48.15 \%$, as shown in Figure 10d. The equivalent circuit models for both geometries have also been investigated and were found to agree well with the simulated responses, as depicted in the figures.

The proposed geometry, owing to its eightfold symmetry, results in a polarization-insensitive behavior, as presented in Figure 11a. The structure is studied for different polarization angles under normal incidence, and the absorptivity remains nearly constant in the range of $8-13.6 \mathrm{GHz}$ for all polarization angles. Furthermore, the design is analyzed under oblique incidence, and revealed that the absorptivity gradually decreases with higher incident angles. The structure can still maintain a $90 \%$ absorptivity in the range of $8.5-11.7 \mathrm{GHz}$ up to $60^{\circ}$ incident angle during $\mathrm{TE}$ polarization. In the $\mathrm{TM}$ mode, the absorptivity reduces at a higher rate with the incident angle and manages to exhibit an absorption bandwidth over the frequency range of $10.5-12.3 \mathrm{GHz}$ at the angle of 


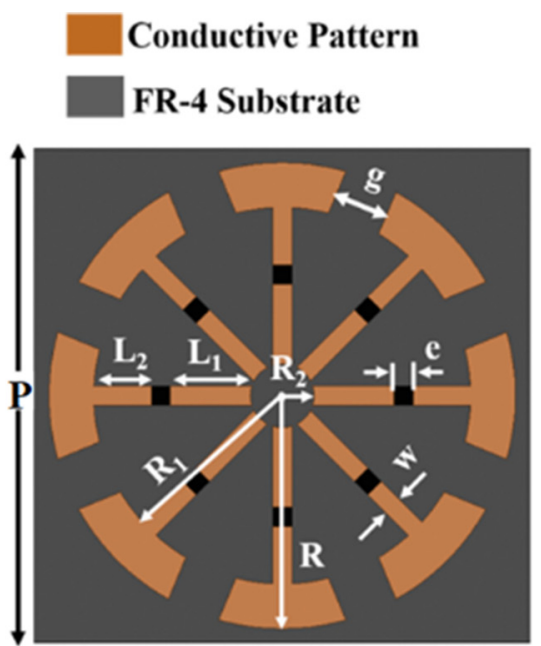

(a)

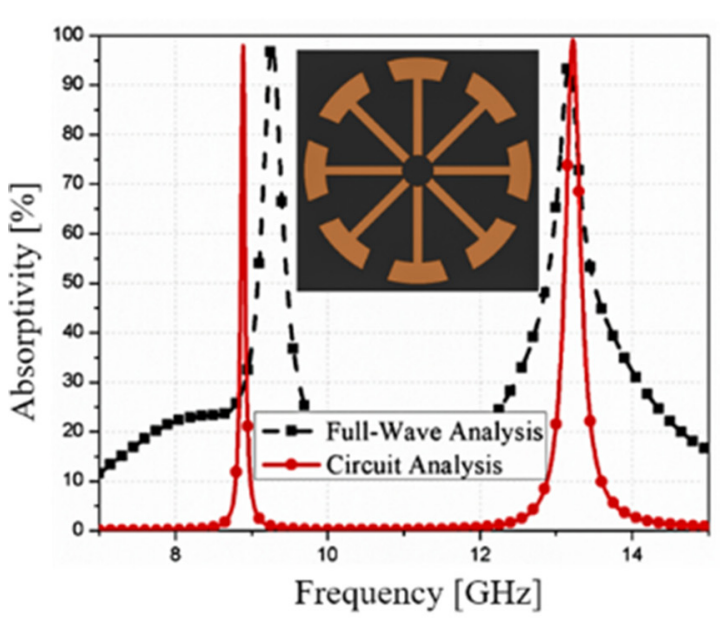

(c)
Eight-Resistive-Arm (ERA)

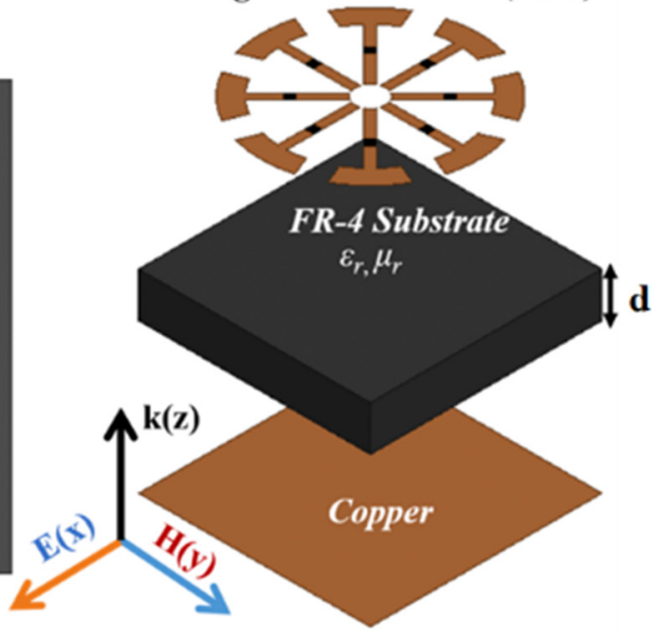

(b)

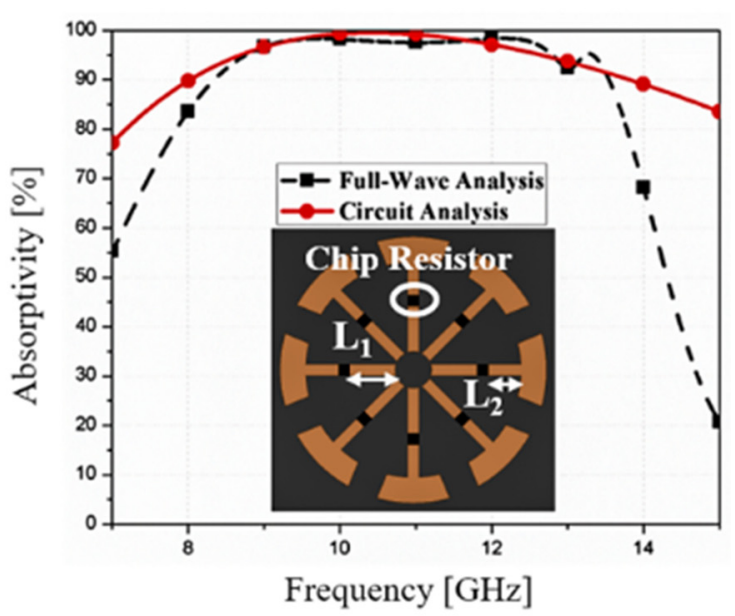

(d)

Fig. 10. Unit cell geometry of the proposed broadband absorber: (a) top view and (b) perspective view. The final geometric dimensions are $P=15.5 \mathrm{~mm}, R=7.3 \mathrm{~mm}, R_{1}=6.1 \mathrm{~mm}, R_{2}=1 \mathrm{~mm}, L_{1}=2.5 \mathrm{~mm}, L_{2}=2 \mathrm{~mm}, w=0.6 \mathrm{~mm}, e=0.6 \mathrm{~mm}, g=1.7 \mathrm{~mm}$, and $d=3 \mathrm{~mm}$. Comparison of the absorptivities obtained from numerical simulation using Ansys HFSS and circuit level simulation: (c) structure without resistors and (d) structure with resistors [58].

incidence $60^{\circ}$. Figures $11 \mathrm{~b}$ and $11 \mathrm{c}$ illustrate the absorption spectra of the proposed geometry for different incident angles under TE and TM polarizations, respectively. It is clearly observed that the proposed design exhibits improved angular stable performances compared to the previous geometry, owing to the increase in the number of resistive arms in the topology.

\section{Conclusions}

We herein presented a compressive study of the recently published narrowband and broadband MM absorbers. The structures described in this article were aimed to exhibit polarization-insensitive and angularly stable absorption responses, unlike most of the earlier reported MM absorbers. Initially, a few narrowband microwave absorbers were illustrated along with their absorption mechanisms. Subsequently, broadband absorbers were discussed in detail. The presented articles are summarized in Table 1 in terms of their absorption characteristic, thickness, polarization insensitivity, and angularly stable behaviors. Owing to their manifold advantages, these structures could be used as potential candidates for various types of absorber applications. The proposed geometries could also be explored to realize reconfigurable absorbers with multifunctional responses.

This work was supported by the National Research Foundation of Korea (NRF) grant funded by the Korea government (MSIP) (No. 2017R1A2B3003856). 


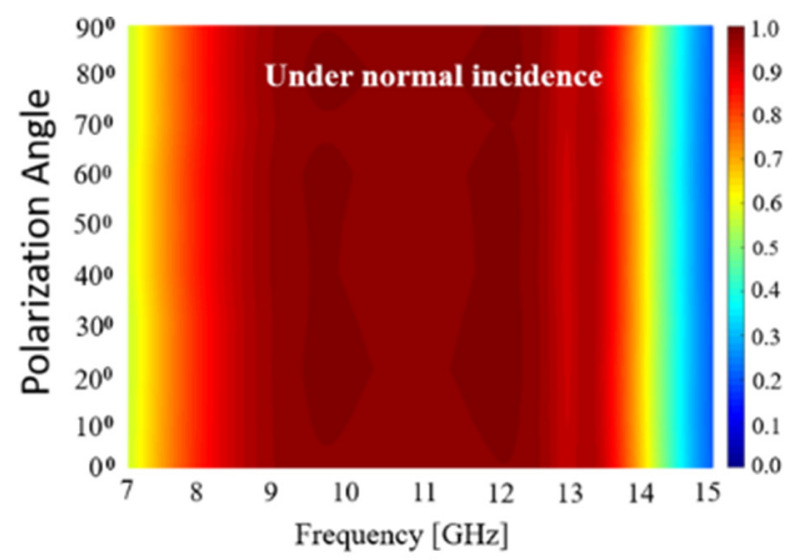

(a)

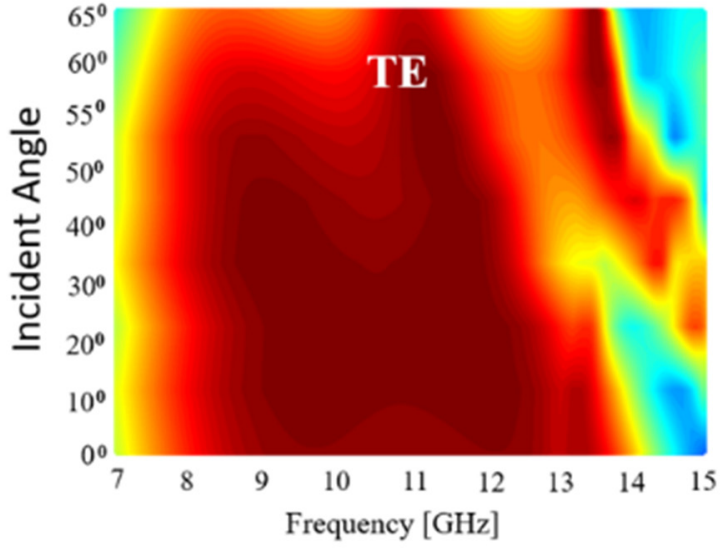

(b)

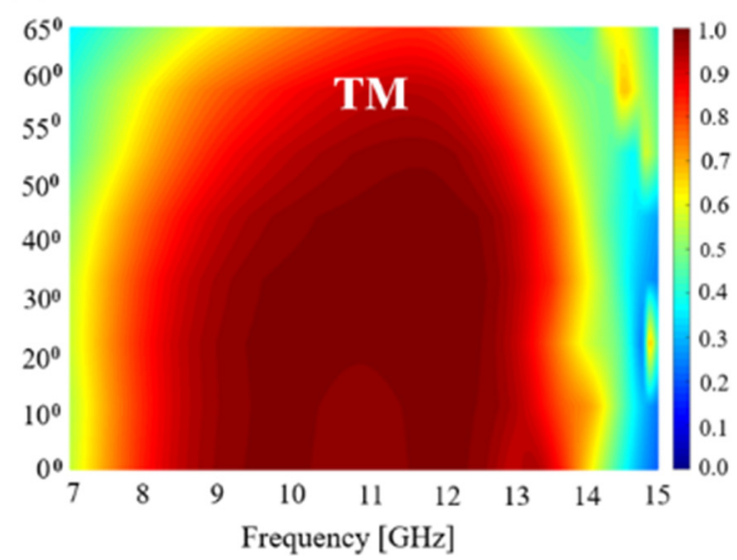

(c)

Fig. 11. Simulated absorption spectra of the proposed broadband absorber (a) for different polarization angles under normal incidence, and for different incident angles under (b) TE polarization and (c) TM polarization [58].

Table 1. Summary of performances of presented MM absorbers.

\begin{tabular}{|c|c|c|c|c|c|c|}
\hline Structure & $\begin{array}{l}\text { Nature of } \\
\text { absorption }\end{array}$ & $\begin{array}{l}\text { Thickness } \\
(\mathrm{mm})\end{array}$ & $\begin{array}{l}\text { Polarization- } \\
\text { insensitive }\end{array}$ & $\begin{array}{l}\text { Peak } \\
\text { absorptivity } \\
\text { at normal } \\
\text { incidence }\end{array}$ & $\begin{array}{l}\text { Absorption } \\
\text { at TE mode }\end{array}$ & $\begin{array}{l}\text { Absorption at } \\
\text { TM mode }\end{array}$ \\
\hline Ref. [31] & Narrowband & 0.6 & Yes & $91 \%$ at $10.44 \mathrm{GHz}$ & $>90 \%$ at $70^{\circ} ; \Delta f<0.96 \%$ & $>90 \%$ at $70^{\circ} ; \Delta f<0.86 \%$ \\
\hline Ref. [35] & Narrowband & 0.8 & Yes & $91.48 \%$ at $10.05 \mathrm{GHz}$ & $>90 \%$ at $70^{\circ} ; \Delta f<1.2 \%$ & $>90 \%$ at $70^{\circ} ; \Delta f \sim 9.45 \%$ \\
\hline Ref. [37] & Narrowband & 0.8 & Yes & $98 \%$ at $9.26 \mathrm{GHz}$ & $>91 \%$ at $70^{\circ} ; \Delta f<0.7 \%$ & $>95 \%$ at $70^{\circ} ; \Delta f<0.7 \%$ \\
\hline Ref. [42] & Narrowband & 0.56 & Yes & $97.5 \%$ at $10.28 \mathrm{GHz}$ & $>90 \%$ at $40^{\circ}$ & $>90 \%$ at $60^{\circ}$ \\
\hline Ref. [45] & BW-enhanced & 0.8 & Yes & $>90 \%$ at $4.34 \mathrm{GHz}$ & $>90 \%$ at $70^{\circ}$ for $1.95 \mathrm{GHz}$ & $>90 \%$ at $70^{\circ}$ for $3.88 \mathrm{GHz}$ \\
\hline Ref. [52] & Broadband & 3.4 & Yes & $>90 \%$ at $6.5 \mathrm{GHz}$ & $>90 \%$ at $40^{\circ}$ for $5.8 \mathrm{GHz}$ & $>90 \%$ at $50^{\circ}$ for $2.3 \mathrm{GHz}$ \\
\hline Ref. [58] & Broadband & 3 & Yes & $>90 \%$ at $5.2 \mathrm{GHz}$ & $>90 \%$ at $60^{\circ}$ for $3.2 \mathrm{GHz}$ & $>90 \%$ at $60^{\circ}$ for $1.8 \mathrm{GHz}$ \\
\hline
\end{tabular}

\section{References}

1. C. Caloz, T. Itoh, Electromagnetic metamaterials, transmission line theory and microwave applications, 1st ed. (John Wiley \& Sons, Inc., New York, 2006)
2. N. Engheta, R.W. Ziolkowski, Metamaterials: physics and engineering explorations, 1st ed. (John Wiley \& Sons, Inc., New York, 2006)

3. J.B. Pendry, A.J. Holden, W.J. Stewart, I. Youngs, Extremely low frequency plasmons in metallic mesostructure, Phys. Rev. Lett. 76, 4773 (1996) 
4. J.B. Pendry, A.J. Holden, D.J. Robbins, W.J. Stewart, Magnetism from conductors and enhanced nonlinear phenomena, IEEE Trans. Microw. Theory Tech. 47, 2075 (1999)

5. D.R. Smith, W.J. Padilla, D.C. Vier, S.C. Nemat-Nasser, S. Schultz, Composite medium with simultaneously negative permeability and permittivity, Phys. Rev. Lett. 84, 4184 (2000)

6. N. Fang, H. Lee, C. Sun, X. Zhang, Sub-diffraction-limited optical imaging with a silver superlens, Science 308, 534 (2005)

7. Y. Dong, T. Itoh, Metamaterial-based antennas, Proc. IEEE 100, $2271(2012)$

8. J. Bonache, I. Gil, J. Garcia-Garcia, F. Martin, Novel microstrip bandpass filters based on complementary split-ring resonators, IEEE Trans. Microw. Theory Tech. 54, 265 (2006)

9. D. Schurig et al., Metamaterial electromagnetic cloak at microwave frequencies, Science 314, 977 (2006)

10. N.I. Landy, S. Sajuyigbe, J.J. Mock, D.R. Smith, W.J. Padilla, Perfect metamaterial absorber, Phys. Rev. Lett. 100, 207402 (2008)

11. A. Fallahi et al., Thin wideband radar absorbers, IEEE Trans. Antennas Propag. 58, 4051 (2010)

12. H. Wakatsuchi, D.F. Sievenpiper, C. Christopoulos, Designing flexible and versatile metamaterial absorbers, IEEE Electromagn. Compat. Mag. 5, 76 (2016)

13. J.-J. Greffet et al., Coherent emission of light by thermal sources, Nature 416, 61 (2002)

14. J. Rosenberg, R.V. Shenoi, T.E. Vandervelde, S. Krishna, O. Painter, A multispectral and polarization-selective surfaceplasmon resonant midinfrared detector, Appl. Phys. Lett. 95, 161101 (2009)

15. N. Liu, M. Mesch, T. Weiss, M. Hentschel, H. Giessen, Infrared perfect absorber and its application as plasmonic sensor, Nano Lett. 10, 2342 (2010)

16. M. Diem, T. Koschny, C.M. Soukoulis, Wide-angle perfect absorber/thermal emitter in the terahertz regime, Phys. Rev. B 79, 033101 (2009)

17. P.L. Richards, Bolometers for infrared and millimeter waves, J. Appl. Phys. 76, 1 (1994)

18. O. Luukkonen, F. Costa, C.R. Simovski, A. Monorchio, S.A. Tretyakov, A thin electromagnetic absorber for wide incidence angles and both polarizations, IEEE Trans. Antennas Propag. 57, 3119 (2009)

19. H. Tao et al., A metamaterial absorber for the terahertz regime: design, fabrication and characterization, Opt. Exp. 16, $7181(2008)$

20. H. Li et al., Ultrathin multiband gigahertz metamaterial absorbers, J. Appl. Phys. 110, 014909 (2011)

21. D.M. Pozar, Microwave engineering, 2nd ed. (John Wiley \& Sons, Inc., New York, 2008)

22. H.-X. Xu et al., Tripleband polarization-insensitive wideangle ultra-miniature metamaterial transmission line absorber, Phys. Rev. B 86, 205104 (2012)

23. C.G. Hu, X. Li, Q. Feng, X.N. Chen, X.G. Luo, Investigation on the role of the dielectric loss in metamaterial absorber, Opt. Exp. 18, 6598 (2010)

24. J.E. Raynolds, Ohmic loss in frequency-selective surfaces, J. Appl. Phys. 93, 5346 (2003)

25. X. Shen et al., Polarization-independent wide-angle tripleband metamaterial absorber, Opt. Exp. 19, 9401 (2011)

26. L. Li, Y. Yang, C. Liang, A wide-angle polarizationinsensitive ultra-thin metamaterial absorber with three resonant modes, J. Appl. Phys. 110, 063702 (2011)
27. H. Zhai, C. Zhan, Z. Li, C. Liang, A triple-band ultrathin metamaterial absorber with wide-angle and polarization stability, IEEE Antennas Wireless Propag. 14, 241 (2015)

28. J.W. Park et al., Multi-band metamaterial absorber based on the arrangement of donut-type resonators, Opt. Exp. 21, 9691 (2013)

29. S. Bhattacharyya, S. Ghosh, K.V. Srivastava, Equivalent circuit model of an ultra-thin polarization-independent triple band metamaterial absorber, AIP Adv. 4, 097127 (2014)

30. Z. Mao et al., Multi-band polarization-insensitive metamaterial absorber based on Chinese ancient coin-shaped structures, J. Appl. Phys. 115, 204505 (2014)

31. D. Lee, J.G. Hwang, D. Lim, T. Hara, S. Lim, Incident angleand polarization-insensitive metamaterial absorber using circular sectors, Sci. Rep. 6, 27155 (2016)

32. S. Ghosh, K.V. Srivastava, An equivalent circuit model of FSS-based metamaterial absorber using coupled line theory, IEEE Antennas Wireless Propag. Lett. 14, 511 (2015)

33. F. Costa, A. Monorchio, G. Manara, Analysis and design of ultrathin electromagnetic absorbers comprising resistively loaded high impedance surfaces, IEEE Trans. Antennas Propag. 58, 1551 (2010)

34. S.A. Tretyakov, S.I. Maslovski, Thin absorbing structure for all incidence angles based on the use of a high-impedance surface, Microw. Opt. Technol. Lett. 38, 175 (2003)

35. T.T. Nguyen, D. Lee, H.K. Sung, S. Lim, Angle- and polarization-insensitive metamaterial absorber based on vertical and horizontal symmetric slotted sectors, Appl. Opt. 55, 8301 (2016)

36. O. Luukkonen et al., Simple and accurate analytical model of planar grids and high-impedance surfaces comprising metal strips or patches, IEEE Trans. Antennas Propag. 56, 1623 (2008)

37. T.T. Nguyen, S. Lim, Wide incidence angle-insensitive metamaterial absorber for both TE and TM polarization using eight-circular-sector, Sci. Rep. 7, 3204 (2017)

38. B. Sanz-Izquierdo, E.A. Parker, Dual polarized reconfigurable frequency selective surfaces, IEEE Trans. Antennas Propag. 62, 764 (2014)

39. D. Lim, D. Lee, S. Lim, Angle- and polarization-insensitive metamaterial absorber using via array, Sci. Rep. 6, 39686 (2016)

40. Y. Cheng, Y. Nie, R. Gong, Metamaterial absorber and extending absorbance bandwidth based on multi-cross resonators, Appl. Phys. B 111, 483 (2013)

41. S. Ghosh, S. Bhattacharyya, D. Chaurasiya, K.V. Srivastava, Polarisation-insensitive and wide-angle multi-layer metamaterial absorber with variable bandwidths, Electron. Lett. 51, $1050(2015)$

42. M. Yoo, H.K. Kim, S. Lim, Angular- and polarizationinsensitive metamaterial absorber using subwavelength unit cell in multilayer technology, IEEE Antennas Wireless Propag. Lett. 15, 414 (2016)

43. W.F. Bahret, The beginnings of stealth technology, IEEE Trans. Aerosp. Elect. Syst. 29, 1377 (1993)

44. S. Celozzi, R. Araneo, G. Lovat, Electromagnetic shielding, 1st ed. (John Wiley \& Sons, Inc., New York, 2008)

45. J. Lee, S. Lim, Bandwidth-enhanced and polarizationinsensitive metamaterial absorber using double resonance, Electron. Lett. 47, 8 (2011)

46. H. Yang et al., Low RCS metamaterial absorber and extending bandwidth based on electromagnetic resonances, Prog. Electromagn. Res. M 33, 31 (2013) 
47. S. Bhattacharyya, S. Ghosh, D. Chaurasiya, K.V. Srivastava, Bandwidth-enhanced dual-band dual-layer polarizationindependent ultra-thin metamaterial absorber, Appl. Phys. A 118, 207 (2015)

48. J. Lee, M. Yoo, S. Lim, A study of ultra-thin single-layer frequency selective surface microwave absorbers with three different bandwidths using double resonance, IEEE Trans. Antennas Propag. 63, 221 (2015)

49. S. Ghosh, S. Bhattacharyya, K.V. Srivastava, Bandwidthenhancement of an ultrathin polarization insensitive metamaterial absorber, Microw. Opt. Technol. Lett. 56, 350 (2014)

50. D. Kundu, A. Mohan, A. Chakraborty, Ultrathin polarization independent absorber with enhanced bandwidth by incorporating giusepe peano fractal in square ring, Microw. Opt. Technol. Lett. 57, 1072 (2015)

51. T.T. Nguyen, S. Lim, Bandwidth-enhanced and wide-angleof-incidence metamaterial absorber using a hybrid unit cell, Sci. Rep. 7, 4814 (2017)

52. T.T. Nguyen, S. Lim, Angle- and polarization-insensitive broadband metamaterial absorber using resistive fan-shaped resonators, Appl. Phys. Lett. 112, 021605 (2018)
53. B.A. Munk, Frequency selective surfaces: theory and design, 1st ed. (John Wiley \& Sons, Inc., New York, 2000)

54. Y. Shang, Z. Shen, S. Xiao, On the design of single-layer circuit analog absorber using double-square-loop array, IEEE Trans. Antennas Propag. 61, 6022 (2013)

55. M. Yoo, S. Lim, Polarization-independent and ultrawideband metamaterial absorber using a hexagonal artificial impedance surface and a resistor-capacitor layer, IEEE Trans. Antennas Propag. 62, 2652 (2014)

56. B.K. Kim, B. Lee, Design of metamaterial-inspired wideband absorber at X-Band adopting trumpet structures, KIEES J. Electromagn. Eng. Sci. 14, 314 (2014)

57. S. Ghosh, S. Bhattacharyya, K.V. Srivastava, Design, characterisation and fabrication of a broadband polarisation-insensitive multi-layer circuit analogue absorber, IET Microw. Antennas Propag. 10, 850 (2016)

58. T.T. Nguyen, S. Lim, Design of metamaterial absorber using eight-resistive-arm cell for simultaneous broadband and wide-incidence-angle absorption, Sci. Rep. 8, 6633 (2018)

Cite this article as: Saptarshi Ghosh, Toan Trung Nguyen, Sungjoon Lim, Recent progress in angle-insensitive narrowband and broadband metamaterial absorbers, EPJ Appl. Metamat. 6, 12 (2019) 